\title{
Crescimento e composição corporal de uma coorte de crianças e adolescentes com diabetes tipo 1
}

\author{
Growth and body composition of a cohort of children \\ and adolescents with type 1 diabetes
}

Maria Fernanda Vanti Macedo Paulino', Sofia Helena Valente de

Lemos-Marini', Gil Guerra-Júnior', André Moreno Morcillo²

\begin{abstract}
RESUMO
Objetivo: Avaliar o crescimento e a composição corporal de crianças e adolescentes com diabetes tipo 1 (DM1). Sujeitos e métodos: Estudo de uma coorte de 44 pacientes com DM1 acompanhados em média por quatro anos, comparados a um grupo controle. Avaliaram-se peso, estatura, índice de massa corporal (IMC), porcentagem de massa gorda (\%MG), índice de massa gorda, cintura e razão cintura/estatura. Resulttados: No sexo feminino, na primeira avaliação a \%MG das pacientes foi menor, enquanto na segunda avaliação a média da cintura das pacientes foi maior que a dos controles. No sexo masculino, a estatura dos pacientes foi menor na primeira avaliação, enquanto o IMC foi maior na segunda. Não foram observadas diferenças entre as variações dos escores z de estatura, peso e IMC e da \%MG ou na distribuição desses escores entre as duas avaliações em ambos os grupos. A análise multivariada mostrou diferença no IMC e na razão cintura/estatura dos dois sexos e também na cintura das meninas. Conclusão: Os pacientes apresentaram crescimento adequado, porém diferiram na composição corporal durante o período do estudo. Arq Bras Endocrinol Metab. 2013;57(8):623-31
\end{abstract}

\section{Descritores}

Diabetes melito tipo 1; criança; adolescente; crescimento; composição corporal

\begin{abstract}
Objective: To evaluate the growth and body composition of children and adolescents with type 1 diabetes mellitus (T1DM). Subjects and methods: A cohort of 44 patients with T1DM were followed up for approximately four years and compared with a control group. Weight, height, body mass index (BMI), body fat percentage (BF\%), fat mass index, waist circumference (WC) and waist-height ratio were determined. Results: In females, in the first evaluation, $\mathrm{BF} \%$ was lower in patients than in controls, while in the second evaluation, mean WC was higher in patients than in controls. In males, height of the patients was lower in the first evaluation, while body mass index (BMI) was higher in the second one. We did not find any differences among the changes in height, weight and BMI z-scores and $\mathrm{BF} \%$ or in the distribution of those z-scores between the two evaluations, in both groups. Multiple regression analysis found differences in BMI and waist-height ratio in both sexes and also in WC in females. Conclusion: The patients had adequate growth but showed discrepancy in their body composition during the study. Arq Bras Endocrinol Metab. 2013;57(8):623-31
\end{abstract}

Keywords

Diabetes mellitus, type 1; child; adolescent; growth; body composition
1 Unidade de Endocrinologia, Departamento de Pediatria, Faculdade de Ciências Médicas e Hospital de Clínicas, Universidade Estadual de Campinas (FCM-HCUnicamp), Campinas, SP, Brasil ${ }^{2}$ Unidade de Crescimento e Desenvolvimento, Departamento de Pediatria, FCM-HC-Unicamp, Campinas, SP, Brasil
Correspondência para:

Maria Fernanda Vanti Macedo Paulino Rua Antônio Augusto de Almeida, 182 Cidade Universitária 13083-755 - Campinas, SP, Brasil rspaulino@hotmail.com

Recebido em 27/Set/2012 Aceito em 29/Maio/2013

\section{INTRODUÇÃO}

$\mathrm{N}^{2}$ as últimas décadas, o maior acesso às insulinas e a liberação da ingesta calórica modificaram o prognóstico de crescimento das crianças com diabetes tipo 1 (DMl). Embora, hoje, a maioria das crianças com DMl atinja estatura final normal, excessivo ganho de peso tem sido frequentemente observado, o que pode resultar em aumento do risco de doenças cardiovasculares (1).

Os efeitos do DMl no crescimento e desenvolvimento puberal de crianças permanecem controversos. Ainda não está claro se o crescimento das crianças diabéticas é influenciado pela duração da doença $(2,3)$, idade de início (3-7), estatura ao diagnóstico (6), grau 
de controle metabólico (3,7-11) ou outros fatores adicionais (1,11-16).

Tendência ao sobrepeso, mais evidente na puberdade, também tem sido relatada, especialmente no sexo feminino $(2,3,6-8,15,17,18)$. A intensificação da insulinoterapia pode resultar em resistência à leptina $\mathrm{e}$ excessivo ganho de massa gorda, particularmente em meninas, o que possibilita problemas de aderência ao tratamento e aumenta o risco de complicações micro e macrovasculares (1).

Em estudo realizado anteriormente, comparando pacientes portadores de DMl pré-púberes e um grupo controle, observamos que as crianças diabéticas eram mais baixas que os controles, embora suas estaturas ainda estivessem dentro dos padrões de normalidade. Não pudemos demonstrar a influência do controle metabólico nesses resultados, uma vez que a maioria das crianças estudadas apresentava controle insatisfatório (19).

O objetivo do presente trabalho foi avaliar o crescimento e a composição corporal de pacientes com diabetes melito tipo 1, utilizando dados de uma coorte acompanhada no Hospital de Clínicas da Universidade Estadual de Campinas.

\section{SUJEITOS E MÉTODOS}

Todas as crianças com $\mathrm{DMl}$ pré-púberes que estavam em acompanhamento no Hospital de Clínicas da Universidade Estadual de Campinas, por ocasião da primeira avaliação, foram convidadas a participar da pesquisa, desde que não tivessem qualquer outra patologia que pudesse interferir no seu crescimento. Entre as que concordaram, $44(23 \mathrm{M} ; 2 \mathrm{lF})$ permaneceram ao longo de todo estudo e compuseram nossa coorte para avaliação do crescimento e composição corporal. A idade por ocasião da primeira avaliação $(2002 / 2004)$ variou de 1,2 a 11,5 anos $(6,9 \pm 2,4$; média \pm desvio-padrão), enquanto na segunda avaliação $(2006 / 2008)$ a idade variou de 4,8 a 15,7 anos $(10,9 \pm 2,3)$. A mediana do tempo de acompanhamento foi de 4,0 anos $(2,9-5,0)$.

O grupo controle na primeira avaliação foi constituído de 66 crianças $(35 \mathrm{M} ; 31 \mathrm{~F})$, com idades entre 1,2 e 11,7 anos. Dessas, 29 (18M; 11F) permaneceram no estudo e foram reavaliadas no segundo exame. Novos sujeitos foram incluídos, totalizando 55 controles $(28 \mathrm{M} ; 27 \mathrm{~F})$ na segunda avaliação, com idades variando de 3,5 a 15,9 anos. Os grupos controle foram compostos por crianças e adolescentes saudáveis, irmãos ou amigos dos pacientes ou ainda outros sujeitos que fo- ram convidados e aceitaram participar do estudo. Não houve pareamento entre os grupos.

Por meio de um questionário, foram obtidas informações referentes à renda familiar per capita (salário-mínimo), escolaridade da mãe (anos) e estatura dos pais.

Dos prontuários dos pacientes, foram obtidos a idade na primeira consulta no serviço (anos), o tempo de doença (anos), a dose de insulina ( $\mathrm{U} / \mathrm{kg} / \mathrm{dia})$ e as hemoglobinas glicadas $(\mathrm{HbAlc})$ realizadas no laboratório do HC-Unicamp no último ano de acompanhamento, para cálculo da média, quando havia duas ou mais determinações, e avaliação do controle metabólico. No serviço, a HbAlc é determinada por cromatografia líquida de alta performance (HPLC).

O exame antropométrico foi realizado por um único examinador, que avaliou o peso, a estatura e a circunferência da cintura de acordo com as recomendações de Lohman e cols. (20). A circunferência da cintura natural foi medida somente na segunda avaliação, com uma fita métrica inelástica, na menor circunferência observada entre o rebordo costal e a crista ilíaca. No estadiamento puberal empregaram-se os critérios propostos por Marshall e Tanner $(21,22)$.

Determinaram-se a resistência e a capacitância com um aparelho de bioimpedância tetrapolar (BIA 101-Q; RJL Systems, Detroit, USA). Na determinação da massa magra (MM) usou-se a equação proposta por Schaefer e cols. (23): massa magra $(\mathrm{kg})=0,65 x$ (estatura ${ }^{2} /$ impedân $^{-}$ cia) $+0,68 \times i d a d e+0,15$. Com base na MM e no peso, determinou-se a porcentagem da massa gorda (\%MG).

Determinou-se a idade óssea dos pacientes pelo método TW2 (método 20 ossos) (24), avaliando-se a última radiografia de punho realizada no serviço, próxima à segunda avaliação.

Calculou-se o índice de massa corporal (IMC) pela fórmula: $I M C=$ peso/estatura ${ }^{2}$. Com base na estatura dos pais determinou-se o alvo parental (25): no sexo masculino: $m e ́ d i a ~=($ estatura pai + estatura $m \tilde{a} e+13) / 2$ e no sexo feminino: $m e ́ d i a=$ (estatura pai + estatura $m \tilde{a} e-13) / 2$. Em ambos os casos foi considerado limite da normalidade a média $\pm 8,5 \mathrm{~cm}$.

Determinaram-se o índice de massa gorda $\left(\mathrm{kg} / \mathrm{m}^{2}\right)$ : $I M G=M G(\mathrm{~kg}) /$ estatura $^{2}(\mathrm{~m})(26)$ e a razão cintura/ estatura (27).

Com relação ao estadiamento puberal (EP), os indivíduos foram divididos em três grupos:

- EPl: pré-púberes.

- EP2: início de desenvolvimento puberal. No sexo feminino: desenvolvimento mamário e/ou pubarca, 
porém sem ter ocorrido a menarca; no sexo masculino: volume testicular maior que $4 \mathrm{~cm}^{3} \mathrm{e}$ menor que $15 \mathrm{~cm}^{3}$.

- EP3: ocorrência de menarca no sexo feminino e volume testicular maior que $15 \mathrm{~cm}^{3}$ no sexo masculino.

Os dados de peso, estatura e IMC foram transformados em escores $\mathrm{z}$, tendo como referencial a curva do CDC 2000 (28), enquanto para a circunferência da cintura empregou-se a curva de McCarthy e cols. (29).

Determinou-se a diferença $(\Delta)$ entre os escores $\mathrm{z}$ da segunda e da primeira avaliação da estatura, do peso, do IMC e da \%MG.

No processamento dos dados, empregou-se o software SPSS versão 16.0 (SPSS Inc, Chicago, IL, USA).

Os dados qualitativos são apresentados em tabelas de contingência, enquanto os quantitativos em tabelas contendo a mediana, a média e o desvio-padrão. Para avaliação da associação entre variáveis qualitativas, empregou-se o teste do qui-quadrado. No caso das variáveis quantitativas, para comparação das médias de dois grupos independentes, empregou-se o teste $t$ de Student ou o teste de Mann-Whitney quando os dados não tinham distribuição normal.

Para análise multivariada, empregou-se a regressão linear múltipla, método stepwise. No modelo da primeira avaliação antropométrica, foram incluídas as variáveis preditoras idade, renda familiar per capita, escolaridade materna e caso (paciente ou controle). Na segunda avaliação, além dessas variáveis, incluiu-se também o desenvolvimento puberal. As variáveis preditoras qualitativas foram transformadas em variáveis "dummy". A variável IMG foi log transformada. Selecionou-se o modelo com o maior coeficiente de determinação ajustado $\left(\mathrm{R}_{\mathrm{aj}}^{2}\right)$, cujos resíduos tivessem média zero e distribuição normal.

Em todos os casos adotou-se o nível de significância de $5 \%$.

O estudo foi aprovado pelo Comitê de Ética em Pesquisa da Faculdade de Ciências Médicas da Universidade Estadual de Campinas (protocolo 776/2006). Os responsáveis pelos indivíduos que compuseram os grupos de estudo (casos e controles) assinaram um Termo de Consentimento Livre e Esclarecido.

\section{RESULTADOS}

Na tabela 1 são apresentadas as características dos pacientes e dos controles em relação a sexo, idade no momento das duas avaliações, renda e escolaridade materna. Nas duas avaliações, houve maior proporção de indivíduos de famílias com maior renda e maior escolaridade no grupo controle. Não houve diferença entre as idades dos pacientes e controles na primeira avaliação $(\mathrm{p}=0,634)$, ao contrário do que ocorreu na segunda avaliação $(\mathrm{p}=0,035)$.

Tabela 1. Distribuição dos pacientes e controles em relação ao sexo, idade, renda familiar per capita e escolaridade da mãe

\begin{tabular}{|c|c|c|c|c|}
\hline & \multicolumn{2}{|c|}{$1^{\text {a }}$ Avaliação } & \multicolumn{2}{|c|}{ 2a Avaliação } \\
\hline & Pacientes & Controles & Pacientes & Controles \\
\hline \multicolumn{5}{|l|}{ Sexo } \\
\hline Masculino & $23(52,2)$ & $35(53,0)$ & $23(52,3)$ & $28(50,9)$ \\
\hline \multirow[t]{2}{*}{ Feminino } & $21(47,7)$ & $31(47,0)$ & $21(47,7)$ & $27(49,1)$ \\
\hline & \multicolumn{2}{|c|}{$p=0,938$} & \multicolumn{2}{|c|}{$p=0,893$} \\
\hline \multirow[t]{3}{*}{ Idade (anos) } & $6,9 \pm 2,4$ & $6,6 \pm 2,7$ & $10,9 \pm 2,5$ & $9,6 \pm 3,2$ \\
\hline & {$[1,2-11,5]$} & {$[1,2-11,7]$} & {$[4,8-15,7]$} & {$[3,5-15,9]$} \\
\hline & \multicolumn{2}{|c|}{$p^{*}=0,634$} & \multicolumn{2}{|c|}{$p^{\star}=0,035$} \\
\hline \multicolumn{5}{|c|}{ Renda per capita (SM) } \\
\hline$<0,5$ & $17(38,6)$ & $6(9,1)$ & $18(40,9)$ & $16(29,1)$ \\
\hline $0,5 \mid---1,0$ & $18(40,9)$ & $5(7,6)$ & $17(38,6)$ & $10(18,2)$ \\
\hline $1,0|---| 2,0$ & $8(18,2)$ & $24(36,4)$ & $8(18,2)$ & $10(18,2)$ \\
\hline \multirow[t]{2}{*}{$>2,0$} & $1(2,3)$ & $31(47,0)$ & $1(2,3)$ & $19(34,5)$ \\
\hline & \multicolumn{2}{|c|}{$p<0,001$} & \multicolumn{2}{|c|}{$p=0,001$} \\
\hline \multicolumn{5}{|c|}{ Escolaridade da mãe (anos) } \\
\hline$\leq 8$ & $31(70,4)$ & $27(40,9)$ & $31(70,5)$ & $22(40,0)$ \\
\hline $9|---| 11$ & $12(27,3)$ & $19(28,7)$ & $12(27,3)$ & $14(25,5)$ \\
\hline \multirow[t]{2}{*}{$\geq 12$} & $1(2,3)$ & $20(30,3)$ & $1(2,3)$ & $19(34,5)$ \\
\hline & \multicolumn{2}{|c|}{$p<0,001$} & \multicolumn{2}{|c|}{$p<0,001$} \\
\hline
\end{tabular}

N (\%); SM: salário mínimo; média \pm desvio-padrão [mínimo - máximo]; $\mathrm{p}^{\star}=$ probabilidade do teste t; $\mathrm{p}=$ probabilidade do teste do qui-quadrado. 
Na tabela 2 são apresentadas as características clínicas e laboratoriais dos pacientes diabéticos, em relação ao sexo. A hemoglobina glicada apresentou-se superior aos níveis preconizados pela Sociedade Americana de Diabetes (30): em 66,7\% na primeira avaliação e em $69,6 \%$ na segunda avaliação entre os pacientes do sexo masculino. Entre os pacientes do sexo feminino isso ocorreu em $88,9 \%$ e $81,0 \%$, respectivamente.

Todos os pacientes e controles eram pré-púberes (EPl) na primeira avaliação. Na segunda avaliação, no sexo masculino, $47,8 \%$ dos pacientes eram do grupo EP2 e 8,7\%, do EP3, enquanto entre os controles $39,3 \%$ eram do grupo EP2 e 7,1\%, do EP3 ( $p=0,833$ ). No sexo feminino, $47,6 \%$ e $23,8 \%$ dos pacientes eram, respectivamente, dos grupos EP2 e EP3, enquanto entre os controles $25,9 \%$ e $29,6 \%$, respectivamente, dos $\operatorname{EP2}$ e EP3 $(\mathrm{p}=0,285)$.
A mediana da diferença entre a idade óssea e a cronológica na segunda avaliação foi de $-0,76$ ano $(-0,70$ $\pm 1,02)$ no sexo masculino e de 0,08 ano $(0,14 \pm 0,57)$ no feminino $(\mathrm{p}=0,008)$. No sexo masculino, $13,6 \%$ $(3 / 22)$ tinham atraso da idade óssea, enquanto no sexo feminino isso não ocorreu. Nenhuma criança ou adolescente apresentou avanço da idade óssea.

A média da idade por ocasião da primeira consulta no serviço foi de 5,7 $\pm 1,7$ anos no sexo feminino ( $\mathrm{Ta}$ bela 3) e 4,7 $\pm 2,2$ anos no sexo masculino (Tabela 4 ).

$\mathrm{Na}$ tabela 3 são apresentados os dados antropométricos dos pacientes e dos controles do sexo feminino. $\mathrm{Na}$ primeira avaliação não se observou diferença entre as médias dos escores $\mathrm{z}$ da estatura, do peso e do IMC e do valor de IMG. A média da \%MG das pacientes foi menor que a dos controles $(20,3 \pm 4,3$ vs. $24,1 \pm 6,9$; $\mathrm{p}=0,020)$. Na segunda avaliação não se observou di-

Tabela 2. Características clínicas e laboratoriais dos pacientes

\begin{tabular}{|c|c|c|c|c|}
\hline & \multicolumn{2}{|c|}{ Masculino $(n=23)$} & \multicolumn{2}{|c|}{ Feminino $(n=21)$} \\
\hline & $1^{\mathrm{a}}$ avaliação & $2^{\mathrm{a}}$ avaliação & $1^{\text {a }}$ avaliação & $2^{\mathrm{a}}$ avaliação \\
\hline Idade (anos) & $6,9(6,6 \pm 2,8)$ & $11,1(10,5 \pm 2,9)$ & $7,1(7,2 \pm 2,0)$ & $11,1(11,3 \pm 2,1)$ \\
\hline Tempo de doença (anos) & $1,8(2,3 \pm 2,2)$ & $6,1(6,3 \pm 2,4)$ & $1,7(2,4 \pm 2,2)$ & $5,9(6,4 \pm 2,4)$ \\
\hline Dose de insulina (U/kg/dia) & $0,62(0,63 \pm 0,16)$ & $0,86(0,87 \pm 0,21)$ & $0,68(0,69 \pm 0,21)$ & $0,94(0,93 \pm 0,21)$ \\
\hline $\mathrm{HbA1c}(\%)$ & $9,1(9,2 \pm 1,8)$ & $8,8(9,2 \pm 1,7)$ & $9,9(9,7 \pm 1,6)$ & $9,1(9,4 \pm 1,8)$ \\
\hline \multicolumn{5}{|l|}{ Desenvolvimento puberal } \\
\hline Pré-púbere (EP1) & $23(100,0 \%)$ & $10(43,5 \%)$ & $21(100,0 \%)$ & $6(28,6 \%)$ \\
\hline Puberdade em curso (EP2) & $0(0 \%)$ & $11(47,8 \%)$ & $0(0 \%)$ & $10(47,6 \%)$ \\
\hline Puberdade adiantada (EP3) & $0(0 \%)$ & $2(8,7 \%)$ & $0(0 \%)$ & $5(23,8 \%)$ \\
\hline $\begin{array}{l}\text { Diferença entre a idade óssea e } \\
\text { a cronológica }\end{array}$ & & $-0,76(-0,7 \pm 1,02)$ & & $0,08(0,14 \pm 0,57)$ \\
\hline
\end{tabular}

Mediana (média \pm desvio-padrão).

Tabela 3. Dados antropométricos dos pacientes e controles do sexo feminino

\begin{tabular}{|c|c|c|c|c|c|}
\hline & \multirow{2}{*}{$\begin{array}{c}1^{\text {a }} \text { consulta } \\
\text { Pacientes } \\
(n=21)\end{array}$} & \multicolumn{2}{|c|}{$1^{\text {a }}$ avaliação } & \multicolumn{2}{|c|}{$2^{\mathrm{a}}$ avaliação } \\
\hline & & $\begin{array}{c}\text { Pacientes } \\
(\mathrm{n}=\mathbf{2 1})\end{array}$ & $\begin{array}{l}\text { Controles } \\
(n=31)\end{array}$ & $\begin{array}{c}\text { Pacientes } \\
(\mathrm{n}=\mathbf{2 1})\end{array}$ & $\begin{array}{c}\text { Controles } \\
(n=27)\end{array}$ \\
\hline Idade (anos) & $5,7 \pm 1,7$ & $7,2 \pm 2,0$ & $6,7 \pm 2,5$ & $11,3 \pm 2,1$ & $9,6 \pm 3,3$ \\
\hline Estatura (escore z) & $0,09 \pm 0,74$ & $-0,17 \pm 1,06$ & $0,11 \pm 0,86$ & $-0,30 \pm 1,06$ & $-0,09 \pm 0,98$ \\
\hline Peso (escore z) & $-0,06 \pm 0,67$ & $-0,12 \pm 0,51$ & $-0,02 \pm 0,91$ & $-0,12 \pm 0,75$ & $-0,37 \pm 1,15$ \\
\hline Índice de massa corporal (escore z) & $-0,11 \pm 0,88$ & $0,01 \pm 0,54$ & $-0,03 \pm 0,98$ & $0,07 \pm 0,74$ & $-0,45 \pm 1,24$ \\
\hline Massa gorda (\%) & & $20,3 \pm 4,3^{*}$ & $24,1 \pm 6,9$ & $23,8 \pm 6,7$ & $23,8 \pm 7,4$ \\
\hline Índice de massa gorda (kg/m²) & & $3,2 \pm 0,7$ & $3,9 \pm 1,4$ & $4,4 \pm 1,9$ & $4,1 \pm 1,7$ \\
\hline Circunferência de cintura (cm) & & & & $60,5 \pm 6,0^{\S}$ & $56,1 \pm 7,1$ \\
\hline Circunferência de cintura (escore z) & & & & $0,36 \pm 0,78^{\varepsilon}$ & $-0,28 \pm 1,02$ \\
\hline Razão cintura/estatura & & & & $0,42 \pm 0,03$ & $0,42 \pm 0,04$ \\
\hline
\end{tabular}

Média \pm desvio-padrão; * $-p=0,020 ; \S-p=0,027 ; £-p=0,026$. 
ferença entre as médias de escore $\mathrm{z}$ da estatura, do peso e do IMC, e dos valores da \%MG e do IMG. A média da cintura das pacientes foi maior que a dos controles $(60,5 \pm 6,0$ vs. $56,1 \pm 7,1 ; \mathrm{p}=0,027)$, entretanto não se observou o mesmo com a razão cintura/estatura.

Na tabela 4 são apresentados os dados do sexo masculino. Na primeira avaliação a média dos escores z de estatura dos pacientes foi menor que a do grupo controle $(-0,08 \pm 0,94$ vs. $-0,37 \pm 0,76 ; \mathrm{p}=0,047)$. Não se observou diferença entre as médias dos escores $\mathrm{z}$ do peso $\mathrm{e}$ do IMC e dos valores da \%MG e do IMG. Na segunda avaliação a média dos escores $\mathrm{z}$ do IMC dos pacientes foi maior que a do grupo controle $(0,45 \pm 0,89 v s$. $-0,21$ $\pm 1,03 ; p=0,020)$. Não se observou diferença entre as médias do escore $\mathrm{z}$ de estatura e peso e dos valores da \%MG, do IMG, da cintura e da razão cintura/estatura.

$\mathrm{Na}$ tabela 5 são apresentadas as variações $(\Delta)$ dos escores z da estatura, do peso e do IMC e dos valores da $\% \mathrm{MG}$ entre as duas avaliações. Tanto no sexo masculino quanto no feminino não se observou diferença entre pacientes e controles.
Nenhum paciente apresentou estatura abaixo do limite inferior do alvo parental; quatro pacientes do sexo masculino e dois do feminino apresentaram estatura acima do limite superior do alvo parental, e nenhum deles apresentou idade óssea além da variação normal de dois anos que pudesse caracterizar idade óssea avançada.

A análise multivariada confirmou a diferença entre as médias de \%MG de pacientes e controles do sexo feminino na primeira avaliação e, na segunda avaliação, mostrou que, além da maior média de cintura, as pacientes também apresentavam maior IMC e razão cintura/estatura em relação aos controles (Tabela 6).

A análise multivariada também confirmou a diferença entre as médias dos escores $\mathrm{z}$ da estatura de pacientes e controles do sexo masculino na primeira avaliação. Referente à segunda avaliação, o modelo confirmou que a média dos escores $\mathrm{z}$ do IMC era maior entre os pacientes e ainda mostrou que a média da cintura/ estatura dos pacientes era maior que a dos controles (Tabela 6).

Tabela 4. Dados antropométricos dos pacientes e controles do sexo masculino

\begin{tabular}{|c|c|c|c|c|c|}
\hline & \multirow{2}{*}{$\begin{array}{c}1^{\text {a }} \text { consulta } \\
\text { Pacientes } \\
(n=23)\end{array}$} & \multicolumn{2}{|c|}{$1^{\mathrm{a}}$ avaliação } & \multicolumn{2}{|c|}{$2^{\mathrm{a}}$ avaliação } \\
\hline & & $\begin{array}{c}\text { Pacientes } \\
(\mathrm{n}=23)\end{array}$ & $\begin{array}{c}\text { Controles } \\
(n=35)\end{array}$ & $\begin{array}{c}\text { Pacientes } \\
(n=23)\end{array}$ & $\begin{array}{c}\text { Controles } \\
(\mathrm{n}=\mathbf{2 8})\end{array}$ \\
\hline Idade (anos) & $4,7 \pm 2,2$ & $6,6 \pm 2,8$ & $6,6 \pm 2,8$ & $10,5 \pm 2,9$ & $9,7 \pm 3,1$ \\
\hline Estatura (escore z) & $0,17 \pm 0,73$ & $-0,08 \pm 0,94^{*}$ & $0,37 \pm 0,76$ & $-0,20 \pm 0,75$ & $0,18 \pm 0,77$ \\
\hline Peso (escore z) & $0,16 \pm 0,84$ & $0,25 \pm 1,13$ & $0,16 \pm 0,91$ & $0,20 \pm 0,96$ & $-0,03 \pm 0,87$ \\
\hline Índice de massa corporal (escore z) & $0,01 \pm 1,21$ & $0,46 \pm 1,17$ & $-0,10 \pm 0,97$ & $0,45 \pm 0,89^{\varepsilon}$ & $-0,21 \pm 1,03$ \\
\hline Massa gorda (\%) & & $22,5 \pm 8,0$ & $20,9 \pm 6,3$ & $21,9 \pm 8,7$ & $20,5 \pm 7,1$ \\
\hline Índice de massa gorda $\left(\mathrm{kg} / \mathrm{m}^{2}\right)$ & & $3,9 \pm 1,8$ & $3,4 \pm 1,3$ & $4,2 \pm 2,1$ & $3,6 \pm 1,7$ \\
\hline Circunferência de cintura (cm) & & & & $62,1 \pm 5,6$ & $59,3 \pm 8,3$ \\
\hline Circunferência de cintura (escore z) & & & & $0,57 \pm 1,01$ & $0,30 \pm 1,05$ \\
\hline Razão cintura/estatura & & & & $0,45 \pm 0,04$ & $0,43 \pm 0,03$ \\
\hline
\end{tabular}

Média \pm desvio-padrão; ${ }^{*}-p=0,047 ; £-p=0,020$.

Tabela 5. Variações $(\Delta)$ de estatura, peso, índice de massa corporal e massa gorda

\begin{tabular}{|c|c|c|c|c|c|c|}
\hline & \multicolumn{2}{|c|}{ Masculino } & \multirow{3}{*}{$\mathbf{p}$} & \multicolumn{2}{|c|}{ Feminino } & \multirow{3}{*}{ p } \\
\hline & Pacientes & Controles & & Pacientes & Controles & \\
\hline & $(n=23)$ & $(n=18)$ & & $(n=21)$ & $(n=11)$ & \\
\hline$\Delta$ Estatura (escore z) & $-0,19(-0,11 \pm 0,50)$ & $-0,18(-0,03 \pm 0,67)$ & 0,990 & $-0,17(-0,13 \pm 0,52)$ & $0,00(-0,07 \pm 0,43)$ & 0,662 \\
\hline$\triangle$ Peso (escore z) & $-0,03(-0,06 \pm 0,66)$ & $-0,09(0,04 \pm 0,61)$ & 0,834 & $0,06(0,01 \pm 0,60)$ & $-0,28(-0,19 \pm 0,49)$ & 0,383 \\
\hline$\triangle \mathrm{IMC}$ (escore z) & $-0,12(-0,07 \pm 0,84)$ & $0,13(0,21 \pm 0,76)$ & 0,335 & $0,00(0,05 \pm 0,63)$ & $-0,23(-0,31 \pm 0,62)$ & 0,177 \\
\hline$\triangle M G(\%)$ & $-2,9(-0,6 \pm 8,4)$ & $0,8(1,2 \pm 8,4)$ & 0,528 & $1,6(3,4 \pm 6,7)$ & $2,1(1,5 \pm 7,5)$ & 0,982 \\
\hline
\end{tabular}

Mediana (média \pm desvio-padrão); $\Delta$ : segunda - primeira avaliação; IMC: índice de massa corporal;

MG: massa gorda; $\mathrm{p}$ : probabilidade do teste de Mann-Whitney. 
Tabela 6. Resultado da análise multivariada

\begin{tabular}{|c|c|c|}
\hline \multicolumn{3}{|c|}{ Sexo feminino $1^{\mathrm{a}}$ avaliação ${ }^{\S}$} \\
\hline Massa gorda (\%) & $\mathrm{R}_{\mathrm{aj}}^{2}=8,9 \%$ & $M G \%=24,077-3,747$. Caso \\
\hline \multicolumn{3}{|c|}{ Sexo feminino $2^{\mathrm{a}}$ avaliação ${ }^{£}$} \\
\hline Escore z IMC & $R_{a j}^{2}=16,4 \%$ & $\mathrm{Z} I \mathrm{IMC}=-0,230-0,852 . \mathrm{GEP} 2+0,702$. Caso \\
\hline Cintura $(\mathrm{cm})$ & $R_{a j}^{2}=67,5 \%$ & Cintura $=46,22+0,885$. Idade $+7,145$. GEP3-2,886. Escolaridade $2+3,275$. Caso \\
\hline Cintura/Estatura & $\mathrm{R}_{\mathrm{aj}}^{2}=21,1 \%$ & $\mathrm{C} / \mathrm{E}=0,495-0,009$. Idade $+0,043 . \mathrm{GEP} 3+0,022$. Caso \\
\hline \multicolumn{3}{|c|}{ Sexo masculino $1^{\mathrm{a}}$ avaliação§ } \\
\hline Escore z estatura & $\mathrm{R}_{\mathrm{aj}}^{2}=5,2 \%$ & Z estatura $=0,373-0,457$. Caso \\
\hline \multicolumn{3}{|c|}{ Sexo masculino $2^{\mathrm{a}}$ avaliação ${ }^{\mathfrak{\varepsilon}}$} \\
\hline Escore z IMC & $\mathrm{R}_{\mathrm{aj}}^{2}=14,5 \%$ & Z IMC $=-0,056-0,603$. Renda $1+0,717$. Caso \\
\hline Cintura/Estatura & $\mathrm{R}_{\mathrm{aj}}^{2}=23,7 \%$ & $\mathrm{C} / \mathrm{E}=0,484-0,005$. Idade $+0,019$. Caso \\
\hline
\end{tabular}

$\mathrm{R}_{\mathrm{aj}}^{2}$ - coeficiente de determinação ajustado.

$\S-\mathrm{y}=\mathrm{a} 1$. Idade $+\mathrm{a} 2$. Renda $1+\mathrm{a} 3$. Renda $2+\mathrm{a} 4$. Renda $3+\mathrm{a} 5$. Escolaridade $1+\mathrm{a}$. Escolaridade $2+\mathrm{a}$. Caso

$£-y=a 1$. Idade + a2. Renda $1+a$ a . Renda $2+a 4$. Renda $3+a 5$. Escolaridade $1+a 6$. Escolaridade $2+a 7$. GEP2 + a8. GEP $3+a 9$. Caso

Idade - Idade em anos - Renda 1 (< 0,5 SM) - [1 - Sim; 0 - Não]; Renda 2 (0,5-1,0 SM) - [1 - Sim; 0 - Não]; Renda 3 (1,0-2,0 SM) - [1 - Sim; 0 - Não]; Escolaridade 1 ( 8 anos) - [1 - Sim; 0 - Não]; Escolaridade 2 (9 a 11 anos) - [1 - Sim; 0 - Não]; EP2 (púbere sem menarca ou vol. testicular < $15 \mathrm{~cm}^{3}$ ) - [1 - Sim; 0 - Não]; EP3 (menarca ou vol. testicular > $\left.15 \mathrm{~cm}\right)^{3}$ - [ 1 - Sim; 0 - Não]

Caso - [1 - diabetes; 0 - controle].

\section{DISCUSSÃO}

Nessa coorte pudemos avaliar o crescimento de pacientes portadores de $\mathrm{DMl}$, com instalação da doença antes da puberdade, em dois momentos distintos com intervalo médio de quatro anos, e compará-los com indivíduos saudáveis. Constatamos que o crescimento foi adequado, sem ganho excessivo de peso ou aumento na \%MG durante o período de seguimento, porém observamos diferenças na composição corporal, sobretudo na circunferência da cintura.

Em estudo anterior com esse grupo de pacientes diabéticos, ainda em estágio pré-puberal, observamos que eles eram significativamente mais baixos que as crianças do grupo controle, embora suas estaturas ainda estivessem dentro dos padrões de normalidade (19).

Todas as nossas crianças apresentaram estatura dentro dos padrões de normalidade e adequada ou acima do alvo familiar. Com exceção dos meninos diabéticos na primeira avaliação, não foram observadas diferenças entre os diabéticos de ambos os sexos em relação ao grupo controle nas duas avaliações. Apesar da doença e do menor nível socioeconômico e de escolaridade das mães, o crescimento desses pacientes era normal na primeira avaliação e continuou sendo durante o período avaliado.

No presente estudo todos os pacientes eram pré-púberes ao diagnóstico, com idade óssea dentro da variação normal para a idade, e evoluíram normalmente à puberdade num período médio de quatro anos de ava- liação. No sexo masculino $43,5 \%$ se mantiveram fora de puberdade e os demais ou iniciaram puberdade $(47,8 \%)$ ou já estavam em fase final de puberdade $(8,7 \%)$, enquanto no sexo feminino $28,6 \%$ se mantiveram fora da puberdade, $47,6 \%$ iniciaram a puberdade e $23,8 \%$ já estavam no final da puberdade, confirmando claramente o esperado, ou seja, que as mulheres fazem a puberdade cerca de um a dois anos antes que os homens.

Embora muitos autores acreditem que a instalação precoce do diabetes possa ser um fator adicional de falência do crescimento, essa condição parece não ter influenciado de forma negativa o crescimento deste grupo estudado até o momento. Assim como a influência da idade da instalação da doença, outro ponto em debate é a fase do crescimento mais afetada nos pacientes portadores de diabetes. Comprometimento do crescimento pré-puberal foi observado por alguns $(4,5)$, enquanto prejuízo do estirão puberal foi notado por outros autores $(5,8,9,16,31)$, e novamente isso aparentemente não ocorreu nesse grupo estudado, pois a estatura não estava prejudicada na fase pré-puberal (primeira avaliação) nem na fase puberal para alguns (segunda avaliação).

Muitos estudos têm demonstrado que, mesmo quando constatada perda de estatura, as crianças com DMl atingem estatura final dentro do alvo parental e dentro das curvas de referência da população $(5,7,13,15,16,31,32)$. Estatura dentro do alvo parental ou acima do limite superior dele também foi observada em nossos pacientes. 
O estudo de Meira e cols. (16), realizado em nosso serviço, envolvendo 40 pacientes diabéticos, com controle inadequado da doença e que já apresentavam estatura final, observou prejuízo do $\mathrm{z}$ de estatura final em relação ao z do início do DMl, provavelmente causado por baixa velocidade de crescimento durante a puberdade, que, no entanto, não teve influência importante na estatura final em relação ao alvo parental.

No presente estudo, apesar de a maioria das crianças e adolescentes apresentar controles insatisfatórios da doença, de acordo com o alvo de hemoglobina glicada estabelecido para a respectiva faixa etária $(30)$, não observamos comprometimento estatural clinicamente relevante durante o seguimento, o que pode sugerir pelo menos duas análises, ou a existência de outros fatores envolvidos na falência do crescimento, em consonância com os achados de vários autores $(2,12-14)$ que também não evidenciaram o efeito do controle metabólico sobre o crescimento, ou o fato de que, para haver comprometimento do crescimento e da puberdade, o controle metabólico tenha que ser muito pior.

No estudo de Bognetti e cols. (14) a dose de insulina foi positivamente relacionada à velocidade de crescimento, enquanto para Pitukcheewanont e cols. (12) esta última foi dependente do ganho de peso e dos níveis de IGFl.

Donaghue e cols. (33) avaliaram o crescimento e as mudanças de IMC em crianças diabéticas estratificadas pelo ano de diagnóstico e concluíram que as crianças diagnosticadas após 1991 mostraram melhora no crescimento linear quando comparadas com aquelas diagnosticadas antes de 1990, sem maiores índices de obesidade. Esse estudo sugere que intensificação da insulinoterapia poderia influenciar positivamente o crescimento de crianças com diabetes, porém, apesar da intensificação da terapia insulínica ocorrida, surpreendentemente isso não se refletiu em melhora do controle glicêmico.

Fato semelhante também foi observado em nossos pacientes. A despeito da tendência à intensificação da terapia insulínica, com três ou mais aplicações de insulina e orientações de correções das hiperglicemias, isso ainda não resultou em melhora da HbAlc média. Ainda assim nossos pacientes vêm crescendo normalmente, o que sugere que os esquemas terapêuticos atuais podem estar contribuindo para que o crescimento das nossas crianças não seja afetado, sem, no entanto, haver controle metabólico completo.

Como já dito, talvez o efeito positivo dos modernos regimes de tratamento possa ser um relativo melhor controle metabólico ocasionado por menores variações glicêmicas pós-prandiais e isso possa desempenhar papel na manutenção do crescimento e desenvolvimento normais dessas crianças e adolescentes. Hirsch e Brownlee (34) destacam a importância das oscilações glicêmicas, sugerindo que a variabilidade glicêmica em combinação com a HbAlc seria um indicador mais seguro de controle glicêmico e risco de complicações crônicas que somente a HbAlc.

Contrariando nossos dados, Danne e cols. (8) mostraram que até pequenas alterações no controle metabólico poderiam levar ao comprometimento da estatura em pacientes com diabetes. Outros autores também demonstraram a correlação entre mau controle metabólico e perda estatural (3,7-11), mas não existe ainda um consenso definindo em qual nível de controle metabólico as alterações no crescimento em crianças com DMl ocorreriam.

A comparação do escore $\mathrm{z}$ de estatura, peso e IMC e o cálculo das diferenças das médias de escores $\mathrm{z}$ nas duas avaliações não permitiram a identificação de anormalidades no crescimento dessas crianças e adolescentes durante esse período de seguimento, não evidenciando, portanto, qualquer efeito da duração da doença.

Vários estudos têm demonstrado tendência de ganho de peso comparado à estatura em crianças com DMl quando elas iniciam a puberdade $(2,3,6-8,17,18)$. Progressivo aumento de massa gorda, particularmente em meninas, tem sido demonstrado e parece estar relacionado à dose de insulina, ao número de aplicações e à intensificação da insulinoterapia (1).

A associação entre altas doses de insulina e ganho de peso pode refletir o efeito lipogênico do excesso de insulina presente na circulação desses pacientes inadequadamente controlados (3).

Embora vários trabalhos tenham demonstrado propensão das crianças diabéticas, em especial das meninas, a ganhar peso durante a puberdade $(2,3,6-8,17,18)$, no nosso estudo não se observou essa tendência, talvez refletindo nosso foco na educação alimentar e na importância da atividade física, considerando o maior risco de complicações clínicas associadas ao excesso de peso, além do fato de nossas pacientes não estarem em uso de altas doses de insulina. Outros fatores que podem estar contribuindo para o não ganho exagerado de peso nesse grupo podem ser o contato frequente com os profissionais de saúde, a demonstração das medidas individuais nas curvas de referência e a discussão constante do plano alimentar. 
Danne e cols. (8) creditam o risco aumentado de sobrepeso próximo à puberdade, particularmente durante a terapia intensiva, ao fato de que, sendo a diminuição da sensibilidade insulínica durante a puberdade restrita ao metabolismo de glicose periférica, a hiperinsulinemia compensatória poderia ampliar os efeitos da insulina no metabolismo de gorduras. Além disso, a menor sensibilidade insulínica em adolescentes do sexo feminino poderia explicar por que as meninas com diabetes são especialmente propensas à obesidade.

Ingberg e cols. (18) observaram que o sobrepeso em adolescentes do sexo feminino com DMl é explicado pelo aumento de massa gorda, e o acúmulo de gordura abdominal está associado com inadequado controle glicêmico, aumento da necessidade de insulina e dislipidemia.

A obesidade abdominal tem sido pouco documentada em jovens com DMl. Sabe-se que a gordura abdominal aumenta o risco de doença cardiovascular em adultos, sendo o diabetes isoladamente um fator de maior risco. A razão cintura/estatura e a circunferência da cintura têm sido correlacionadas com a gordura visceral e têm sido consideradas preditoras de risco adicional na morbimortalidade cardiovascular desses pacientes.

Os dados obtidos neste estudo mostraram que a média da circunferência da cintura e da razão cintura/ estatura das diabéticas foi maior que a dos controles, assim como a média da razão cintura/estatura dos pacientes do sexo masculino, mostrando diferenças na composição corporal entre os grupos. Considerando que o aumento de gordura visceral poderia contribuir para um maior risco de desenvolvimento das complicações macrovasculares, como doença arterial coronariana, a importância do estudo da distribuição da gordura, além da gordura corporal total, deve ser enfatizada.

Acreditamos que esses achados possam servir de estímulo para o estudo da obesidade abdominal nesse grupo de pacientes, idealmente envolvendo técnicas de imagem que possam identificar a gordura visceral com mais acurácia. Além disso, uma avaliação por período de tempo maior, até a obtenção da altura final e a investigação de outros fatores de risco agregados, como a determinação da espessura da íntima das carótidas e aorta desses pacientes, por meio de exame ultrassonográfico, seria de grande valia tendo em vista às justificativas já citadas.

Concluindo, este grupo de pacientes com DMl apresentou crescimento adequado, sem tendência ao ganho de peso excessivo, ou tampouco aumento na porcentagem de massa gorda durante o período do estudo. Entretanto, demonstramos diferenças na composição corporal, sobretudo na circunferência da cintura, evidenciando a necessidade de adoção de métodos de avaliação da distribuição da gordura na avaliação do estado nutricional, em especial nesse grupo de pacientes, nos quais a obesidade central está relacionada a maior risco metabólico.

Declaração: os autores declaram não haver conflitos de interesse científico neste estudo.

\section{REFERÊNCIAS}

1. Dunger D, Ahmed L, Ong K. Growth and body composition in type 1 diabetes mellitus. Horm Res. 2002;58:66-71.

2. Luna $R$, Alvarez-Vasquez $P$, Hervás $E$, Casteras $A$, Mendez LP, Páramo $C$, et al.The role of diabetes duration, puberal development and metabolic control in growth in children with type 1 diabetes mellitus. J Pediatr Endocrinol Metab. 2005;18:1425-31.

3. Elamin A, Hussein O, TuvemoT. Growth, puberty, and final height in children with type 1 diabetes. J Diabetes Complications. 2006;20:252-6.

4. Tattersall RB, Pyke DA. Growth in diabetic children: studies in identical twins. Lancet. 1973;17:1105-9.

5. Brown M, Ahmed ML, Clayton KL, Dunger DB. Growth during childhood and final height in type 1 diabetes. Diabet Med. 1994;11:182-7.

6. Fernandes VT, Verreschi ITN, Dib SA. Development according to puberal stage in Brazilian children and adolescents with shortterm diabetes. Braz J Med Biol Res. 2001;34(10):1315-23.

7. Martinez RG, Garcia EG, Gomez MDG, Liorente JLG, Fernandez PG, Perales AB. Talla final em diabéticos tipo 1 diagnosticados em la edad pediátrica. An Pediatr. 2009;70:235-40.

8. Danne T, Kordonouri O, Enders I, Weber B. Factors influencing height and weight development in children with diabetes - Results of the Berlin retinopathy study. Diabetes Care. 1997;20(3):281-5.

9. Ahmed ML, Connors MH, Drayer NM, Jones JS, Dunger DB. Pubertal growth in IDDM is determined by $\mathrm{HbA} 1$ levels, sex, and bone age. Diabetes Care. 1998;21(5):831-5.

10. RodriguesTMB, Silva IN. Estatura final de pacientes com diabetes mellitus tipo 1. Arq Bras Endocrinol Metab. 2001;45:108-14.

11. Li G, Mick G, Wang X, Xue J, McCormick K. Growth hormoneIGF1 axis and growth velocity in Chinese children with type 1 diabetes mellitus. J Pediatr Endocrinol Metab. 2006;19:1313-8.

12. Pitukcheewanont $P$, Alemzadeh $R$, Jacobs WR, Jones BH, Eberle AJ. Does glycemic control affect growth velocity in children with insulin-dependent diabetes mellitus. Acta Diabetol. 1995;32:148-52.

13. Salerno MC, Argenziano A, Di Maio S, Gasparinl N, Formicola S, De Fellipo G, et al. Puberal growth, sexual maturation, and final height in children with IDDM. Diabetes Care. 1997;20(5):721-3.

14. Bognetti E, Riva MC, Bonfanti R, Meschi F, Viscardi M, Chiumello G. Growth changes in children and adolescents with short-term diabetes. Diabetes Care. 1998;21(8):1226-9.

15. Lebl J, Schober E, Zidek T, Baldis S, Rami B, Pruhova S, et al. Growth data in large series of 587 children and adolescents with type 1 diabetes mellitus. Endocr Regul. 2003;37:153-61.

16. Meira SO, Morcillo AM, Lemos-Marini SHV, Paulino MFVM, Minicucci WJ, Guerra-Junior G. Crescimento puberal e altura 
final em 40 pacientes com diabetes mellitus tipo 1. Arq Bras Endocrinol Metab. 2005;49:396-402.

17. Du Caju MVL, Rooman RP, Beeck LO. Longitudinal data on growth and final height in diabetic children. Pediatr Res. 1995;38(4):607-11.

18. Ingberg CM, Särnblad S, Palmer M, Schvarcz E, Berne C, Aman J. Body composition in adolescent girls with type 1 diabetes. Diabet Med. 2003;20(12):1005-11.

19. Paulino MFVM, Lemos-Marini SHV, Guerra-Junior G, Miniccuci WJ, Mendes CT, Morcillo AM. Crescimento e composição corporal de crianças com diabetes mellitus tipo 1. Arq Bras Endocrinol Metab. 2006;50(3):490-8.

20. LohmanTG, RocheAF, Martorell R. Anthropometric standardization reference manual. Illinois: Human Kinetics Books; 1988. p. 90.

21. MarshallWA, Tanner JM. Variations in pattern of pubertal changes in girls. Arch Dis Childh. 1969;44:291-303.

22. Marshall WA, Tanner JM. Variations in the pattern of pubertal changes in boys. Arch Dis Childh. 1970;45:13-23.

23. Schaefer F, Georgi M, Zieger A, Schärer K. Usefulness of bioeletric impedance and skinfold measurements in predicting fat-free mass derived from total body potassium in children. Pediatr Res. 1994;35:617-24.

24. Tanner JM, Whitehouse $\mathrm{RH}$, Cameron N, Marshall WA, Healy MJR, Goldstein H. Assessment of skeletal maturity and prediction of adult height (TW2 Method). 2th ed. Orlando: Academic Press; 1983. p. 108.

25. Tanner JM, Goldstein H, Whitehouse RH. Standards for children's height at ages 2-9 years allowing for height of parents. Arch Dis Chilh. 1970;45:755-62.
26. Schutz Y, Kyle UUG, Pichard C. Fat-free mass index and fat mass index percentiles in Caucasians aged 18-98 y. Int $\mathrm{J}$ Obes. 2002;26:953-60.

27. Li C, Ford ES, Mokdad AH, Cook S. Recent trends in waist circunference and waist-height ratio among US children and adolescents. Pediatrics. 2006;118:1390-8.

28. Kuczmarski RJ, Ogden $C L$, Grummer-Strawn LM, Flegal KM, Mei Z, Guo S, et al. CDC growth charts: United States. Adv Data. 2000;8:1-27.

29. McCarthy HD, Jarret KV, Crawley HF. The development of waist circumference percentiles in British children aged 5.0-16.9 y. Eur J Clinl Nutr. 2001;55:902-7.

30. American Diabetes Association: Standards of Medical Care in Diabetes - 2011. Diabetes Care. 2011;34 (suppl. 1):S39.

31. Salardi S, Tonioli S, Tassoni P, Tellarini M, Mazzanti L, Cacciari E. Growth and growth factors in diabetes mellitus. Arch Dis Child. 1987;62:57-62.

32. Choudhury S, Stutchfield P. Linear growth and weight gain in diabetic children - A cross-sectional and longitudinal evaluation. J Pediatr Endocrinol Metab. 2000;13(5):537-44.

33. Donaghue KC, Kordonori O, Chan A, Silink M. Secular trends in growth in diabetes: are we winning? Arch Dis Child. 2003;88:151-4.

34. Hirsch IB, Brownlee M. Should minimal blood glucose variability become the gold standard of glycemic control? J Diabetes Complications. 2005;19:178-81. 